\title{
EDITORIAL
}

\section{Mentoring the Wisconsin way}

\author{
Whether or not an undergraduate decides to pursue a career in science can often depend \\ on their first experience in the lab, yet the individuals assigned as their mentors are often \\ given no formal training in mentoring.
}

As an undergraduate embarking on your first research project, who showed you how to get started - how to streak a plate, pour an agarose gel or set up an overnight culture? The chances are that it wasn't the lab PI, but a member of the unofficial army of mentors, usually a postdoc.

In many ways this training system is ideal, as experienced postdocs can have an unrivalled knowledge of the necessary techniques, and many postdocs would shudder at the thought of their PI coming into the lab to train students. However, although the additional responsibility is often regarded as an official part of the job, most postdocs receive no formal mentoring training. Additionally, it is not only postdocs who are expected to be mentors - grad students are also often asked to take on an undergraduate student for the summer or for a period during term-time, at what can be a crucial point in their own research, and again usually receive no formal training. So, how can the mentoring experience be improved for both mentors and mentees?

This question was addressed recently in an interesting article published in Science ${ }^{1}$, which analysed the success of a mentoring seminar developed by The Wisconsin Program for Scientific Teaching (WPST) at the University of Wisconsin-Madison (UW-M). The WPST has been at the forefront of revolutionizing teaching in the life sciences, promoting the development and implementation of 'scientific teaching' methods based on active learning strategies ${ }^{2}$. Although applicable to the life sciences in general, the seminar might be of particular interest to microbiologists owing to the involvement of Jo Handelsman, well-known plant pathologist and Howard Hughes Medical Institute (HHMI) Professor in the Department of Plant Pathology at UW-M.

The Wisconsin Mentoring Seminar provides a framework for learning how to become a mentor. It comprises eight 1-hour sessions run by a facilitator, with each session covering a different aspect of effective mentoring, including communication skills, managing expectations, how to identify and effectively resolve problems, and diversity awareness. Facilitators are provided with a manual, which is available online $\mathrm{e}^{3}$ and which contains a discussion outline for each session, including discussion questions, assignments and suggested reading. The seminar is not prescriptive, but encourages mentors to use scientific learning and develop their own mentoring philosophy. Each session is based around discussion questions, and participants are encouraged to discuss their own mentoring experiences, with a view to learning how they might handle similar situations differently in the future.

Over the past two and a half years, the seminar has run on 22 occcasions at 11 research institutes including UW-M and, in the spirit of scientific teaching, which is an iterative process involving evaluation, feedback and revision, the experiences of the facilitators, mentors and mentees involved are now reported ${ }^{1}$.

All of the facilitators surveyed found the manual useful and all would recommend the experience of being a facilitator to colleagues. Trained mentors were more likely to discuss their student's expectations of them as a mentor and consider issues of diversity than were untrained mentors, and $87 \%$ of trained mentors said that they would recommend the course to their peers. The mentees surveyed also reported a positive experience, with students who were assigned a trained mentor more likely to agree with the statement that their mentor "regularly assessed the skills and knowledge that they had gained in the lab" than students with an untrained mentor. Additionally, students who had previously been mentored said that a trained mentor was more available to them and more interested in them as individuals.

A good mentor is constantly learning about their own mentoring style as each mentoring experience is different from the one before. There is no substitute for experience - as stated in the preface to the seminar, "Good mentors discover their own objectives, methods and style by mentoring. And mentoring. And mentoring some more," however, the Wisonsin Mentoring Seminar is an excellent first step towards becoming a good mentor. The first research project can often be 'make or break' for undergraduates, with a good experience likely to encourage them to go on to pursue a career in science, and so we would encourage everyone involved in mentoring to access the manual and think about implementing the programme.

\footnotetext{
Pfund, C. et al. The merits of training mentors. Science 311 473-474 (2006)

2. Handelsman, J. et al. Scientific teaching. Science 304, 521-522 (2004)

3. Handelsman, J. et al. Entering Mentoring: A Seminar to Train a New Generation of Scientists (University of Wisconsin Press, Madison, 2005); available online at < http://www.hhmi.org/grants/pdf/labmgmt/ entering_mentoring.pdf $>$.
} 